\title{
Effects of stimulus site on the pattern of skin conductance responses evoked from spinal man
}

\author{
MARCUS J. FUHRER ${ }^{1}$ \\ From the Departments of Rehabilitation and Psychiatry, Baylor College of Medicine \\ and the Texas Institute for Rehabilitation and Research, Houston, Texas, U.S.A.
}

SYNOPSIS The spatial organization of sudomotor responses mediated by the cervically transected human spinal cord was investigated by recording skin conductance responses from the volar surfaces of the hands and feet of 10 patients after pulse trains applied separately to the skin of each extremity. Each stimulus site tended to be associated with a distinctive pattern of skin conductance responses.

It has been long established from clinical observations that reflex sweating can be mediated by the transected human spinal cord (Head and Riddoch, 1917; Thomas, 1921). By visually monitoring the sweat production of patients with complete section of the cord, it was demonstrated further that spinal reflex sweating can be evoked by an interoceptive stimulus such as distension of the bladder (Guttman and Whitteridge, 1947) as well as by cutaneous stimulation (List and Pimenta, 1944).

The more phasic aspects of reflex spinal sweating have been studied using electrodermal methods to acquire a continuous quantifiable record of sympathetically mediated increases in presecretory glandular activity. It has been established, for example, that both skin conductance responses and skin potential responses can be evoked by cutaneous stimuli applied below the level of transection in the chronic spinal cat (Richter, 1930; Richter and Shaw, 1930; Ladpli, 1962). Sourek $(1964,1965)$ reported that electrocutaneous stimulation applied below the level of a transverse lesion of the human spinal cord was not adequate to evoke skin potential responses from the plantar surfaces. Using higher stimulus intensities, however, Fuhrer and Kilbey (1967) demonstrated that skin conductance responses could be elicited reliably from such patients under otherwise similar conditions.

1 Address for reprints: Texas Institute for Rehabilitation and Research, 1333 Moursund Avenue, Houston, Texas 77025, U.S.A.

(Accepted 11 April 1975.)
A subsequent study (Fuhrer, 1971a) was devoted in part to determining how the occurrence and amplitude of palmar and plantar skin conductance responses varied according to the location and intensity of stimulation applied to patients with a chronic transection of the cervical cord. By means of discrete pulse trains applied percutaneously to the tibial nerve of one leg or the other, a characteristic pattern of responses from the extremities was noted. Evidence for a regional organization of responses, dependent upon the site of stimulation, was reflected by the finding that responsiveness was substantially greater from the stimulated extremity than from the opposite lower extremity. Marked intersegmental irradiation of the afferent stimulation was also observed-for example, bilaterally recorded responses from the palms were of higher amplitude than responses from the plantar surfaces, even though the stimulus was delivered to a peripheral nerve in the lower leg.

The present investigation was designed to provide more comprehensive information regarding effects of the locus of stimulation on the pattern of skin conductance responses mediated by the cervically transected human spinal cord. In addition to stimulating lower extremity sites, pulse trains were applied to upper extremity skin sites innervated by the isolated spinal cord. This made it possible to determine whether stimulating and recording from the same extremity results in a similar regional organization of responsiveness for the upper and lower extremities. It was also 
possible to determine whether intersegmental irradiation of afferent input is as conspicuous when an upper extremity is stimulated as it is when the stimulus is applied to a lower extremity. Reflex movements evoked by stimulating different sites were monitored in order to compare the functional organization of somatic and sudomotor reflex activity.

\section{METHOD}

SUBJECTS The 10 male patients were between 18 and 34 years of age. Each had sustained a traumatic, functionally complete transection of the cervical spinal cord between the fourth and seventh segments. At the time of the study, 15-83 months had passed since injury. Each individual was examined neurologically by at least two examiners before admission to the study, and in no case was voluntary motor functioning or sensation detected below the level of the lesion. All had been discharged from a comprehensive rehabilitation programme and were, with one exception, either employed or attending a university. They were all free of urinary tract infection or decubitus ulcers. All spasmolytic or sympathetically active medication was withdrawn no less than $\mathbf{4 8}$ hours before the study session.

APPARATUS AND PROCEDURE The special-purpose skin conductance monitor (Electronic Laboratories) contained four independent amplifiers, each of which provided $0.5 \mathrm{~V}$ constant-voltage excitation and a capability of recording conductance changes from 0.003 to $9.999 \mu \mathrm{mhos} / \mathrm{cm}^{2}$ from a single recording site. The output of each amplifier was AC- coupled into a six-channel oscillograph (Siemens, Model E) using a four second time constant. Four additional amplifiers provided an indication of the conductance level of each site within the range of 0.25-100.00 $\mu \mathrm{mhos} / \mathrm{cm}^{2}$. Skin conductance levels were monitored visually from a meter provided with a suitable range selector switch. An electrogoniometer providing a $3 \mathrm{~mm}$ deflection for each degree of joint excursion was attached to the right ankle, and in some cases, another unit was applied to the left ankle. The stimulator was a constant-current design (Nuclear Chicago, Model 7150) which supplied a transformer-isolated train of rectangular pulses of electricity.

The temperature in the laboratory was maintained between 22 and $24^{\circ} \mathrm{C}$. Using techniques described previously (Fuhrer, 1971a), active sites for recording skin conductance responses were prepared on the thenar eminence of each palm and on the medial plantar surface of each foot. The reference electrode for each palmar site was located on the dorsal aspect of the forearm, and the reference electrode for each plantar site was positioned on the lateral aspect of the lower leg. Clip-on electrodes for applying electrocutaneous stimulation were the same as used previously (Fuhrer, 1973). Separate pairs of

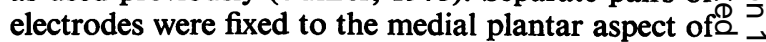
each foot and to the hypothenar eminence of each $>$ palm.

As described in greater detail by Fuhrer (1971b), test was conducted first to discern whether there waso $\overrightarrow{0} \overrightarrow{0}$ any evidence that skin conductance responses from $* \vec{v}$ any of the recording sites were under suprasegmental control. This consisted of six repetitions of a 1000 $\mathrm{Hz}, 100 \mathrm{~dB}, 500 \mathrm{~ms}$ tone presented bilaterally through

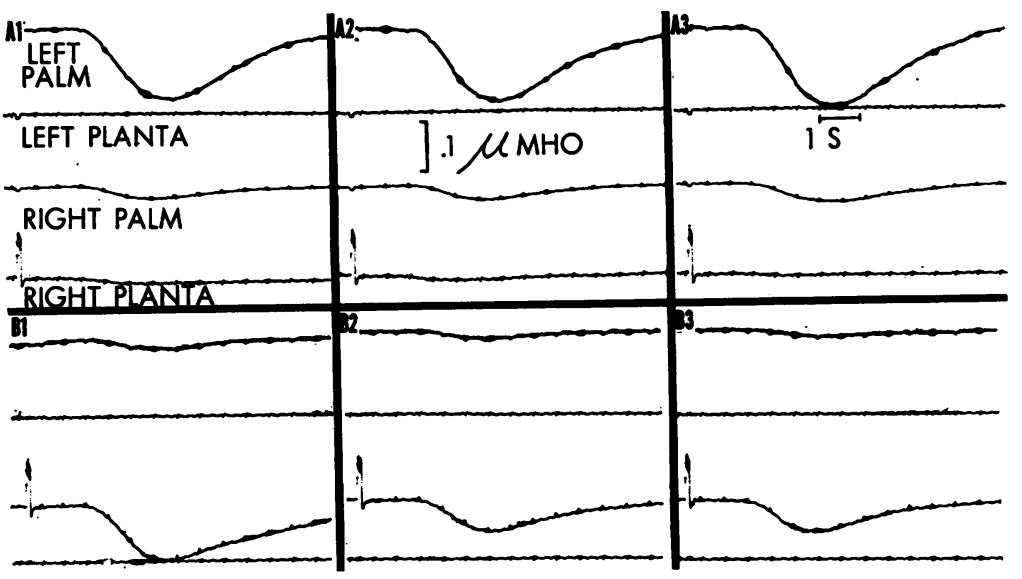

FIC. 1 Panels A1-A3: skin conductance responses from the palms and plantar surfaces evoked by a pulse train applied to the right planta. Delivery of the stimulus is indicated by a transitory upward deflection appearing in the trace recorded from the right planta. Concurrent responses to three successive stimulus applications separated by approximately $20 \mathrm{~s}$ are shown. Panels B1-B3: recordings from the same sites after application of the stimulus to the right palm. 
earphones at roughly 20 second intervals. A workup procedure was then begun to establish the intensity of electrocutaneous stimulation to be used. The stimulator was adjusted to supply a pulse train of $40 \mathrm{~ms}$ duration, a frequency of $100 \mathrm{~Hz}$, and a pulse duration of $1.0 \mathrm{~ms}$. The current level was set initially at $0.1 \mathrm{~mA}$ and a pulse train was applied separately to each of the four sites. Successive sets of four stimuli each were increased in steps of $0.2 \mathrm{~mA}$. The work-up procedure was terminated when the flexor withdrawal activity of a lower extremity was sufficiently violent to disturb the patient or when a current level of $5.0 \mathrm{~mA}$ was reached. This level was the maximum permissible in order to avoid visible damage to the skin. For the group, the final current level ranged between 2.8 and $5.0 \mathrm{~mA}$, the mean being $4.6 \mathrm{~mA}$. Subsequently, the current level which had been established was applied on six occasions to each of the four stimulus sites. The order of the presentations of stimuli was randomized except for the constraint that a particular site should not be stimulated more than twice successively. The interstimulus intervals ranged unsystematically between 20 and 40 seconds. Separate observers visually monitored reflex movements of the upper and lower extremities. A transcript was prepared later from their tape recorded comments, and the content was related to the oscillographically recorded data.

Skin conductance responses were quantified by measuring the deflection to the nearest millimetre from its onset to its peak and then converting this value to micromhos. Based upon results of a previous study (Fuhrer, 1971a), scoring was limited to palmar responses with a latency of 1-3 s and plantar responses with a latency of $2-4 \mathrm{~s}$.

\section{RESULTS}

Since the evidence of the neurological examination suggested that the patients' lesions were rostral to the eighth cervical segment and thus above the sympathetic outflow to the extremities, no systematic association was expected between presentations of the high intensity auditory stimuli and the occurrence of skin conductance responses during the post-stimulus interval. For eight patients, no responses were recorded from any site during any of the post-stimulus intervals. Responses to two of the six tone presentations were recorded from each of the two remaining patients, and in both cases the responses were from the same palmar site. However, since the frequency and amplitude of these responses were equalled or surpassed by responses recorded during stimulus-free intervals equidistant between stimulus presentations, it was concluded that the responses after the auditory stimulus were a form of spontaneous electrodermal activity described by Fuhrer and Kilbey (1967) in such patients.

Representative examples of the difference in the pattern of skin conductance responses evoked by applying the pulse train to two different stimulus sites are contained in Fig. 1. The recordings were obtained from a patient with a transverse lesion at the fifth cervical segment. In panels A1-A3, the stimulus was applied to the plantar aspect of the right foot. A stimulus artefact is observable in the trace recorded from the right planta. The largest amplitude response was recorded from the left palm, and a smaller response was obtained from the right palm. Responsiveness from the lower extremities was limited to a very low amplitude response from the right planta. The recordings depicted in the three panels illustrate the stability of this pattern of responding over the course of three repetitions of the stimulus. Reflex movements were limited to dorsiflexion of the toes and ankle of the right leg, accompanied by slight flexion of the right knee joint.

Panels B1-B3 of Fig. 1 illustrate the pattern of responsiveness obtained when the stimulus was applied to the hypothenar surface of the right palm of the same patient. The response configuration was characterized by the largest response being from the right palm, with a substantially lower amplitude response being recorded from the left palm. No responses were obtained from the plantar surfaces. This pattern was reproduced with considerable fidelity when the stimulus was repeated on three successive occasions. No observable movements of the upper or lower extremities were evoked by stimulating this site.

The median percentage incidences of response from the four sites responding to stimulation applied separately to each extremity are shown in Fig. 2. It may be noted that stimulation of the palms was on no occasion associated with responses from the plantar surfaces. Furthermore, responses occurred more frequently from the stimulated palm than from the opposite palm for all patients.

When stimulation was applied to either one of the lower extremity sites, responses were re- 


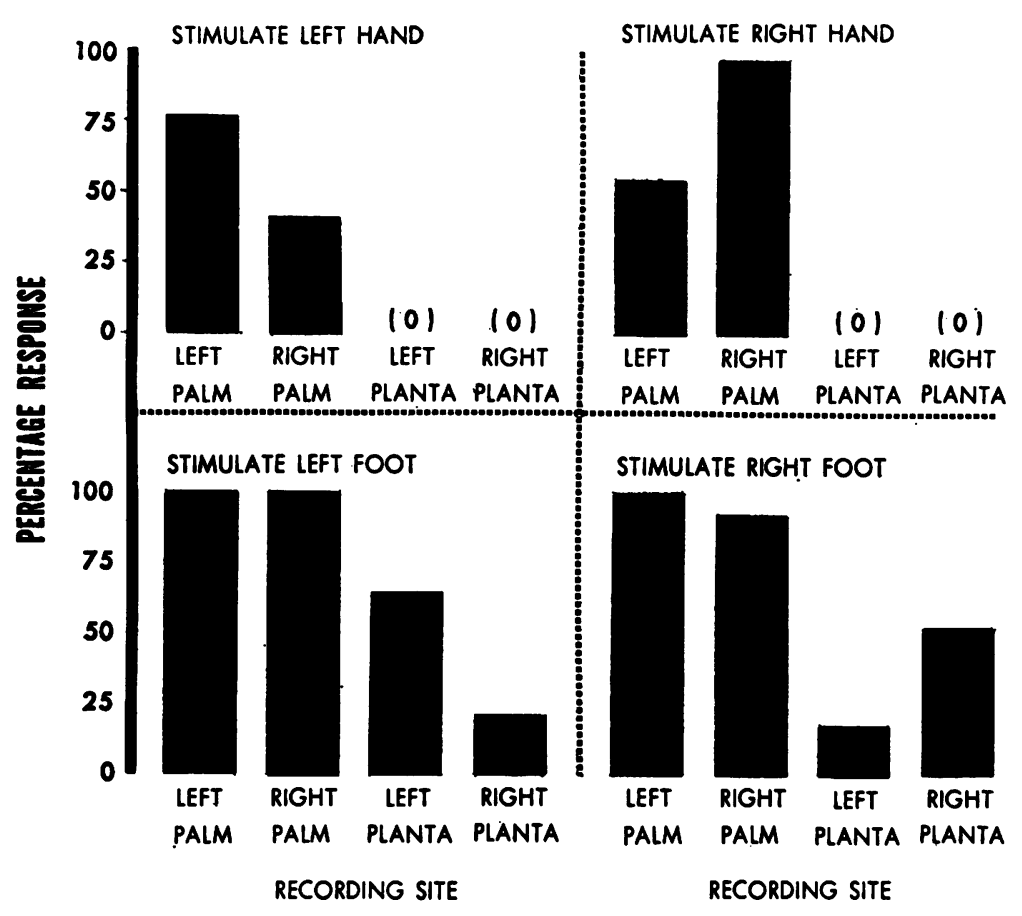

FIG. 2 Median percentage incidences of responses from the four extremities to six repetitions of a pulse train applied separately to the plantar arch of each foot.

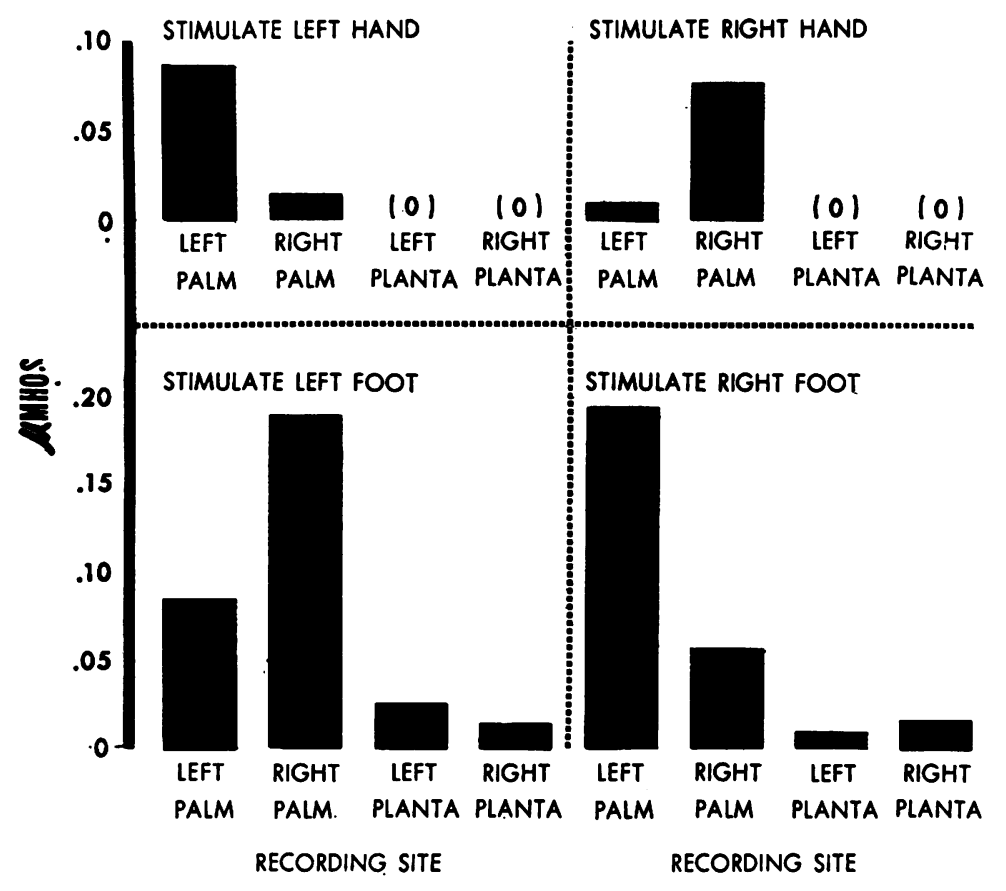

FIG. 3 Median amplitude of responses from the four recording sites to six repetitions of the stimulus applied separately to each of the four extremities. 
corded almost without exception from both palms, though not as consistently from the plantar surfaces. According to results of the Wilcoxon test (Siegel, 1956), there was evidence that the percentage incidence of responding was reliably greater from the plantar surface that was stimulated than from the opposite plantar surface for stimulation of the left planta $(T=1$, $\mathrm{n}=7, \mathrm{P}<0.05)$ and for stimulation of the right planta $(T=0, n=6, P<0.05)$.

Additional effects due to varying the location of the stimulus were revealed by analysing the amplitude of responses recorded concurrently from the four extremities. It should be noted that entries of zero (no response) were omitted from calculating the median amplitude values displayed in Fig. 3. In most respects, the data for response amplitudes paralleled the outcomes reflected in the percentage data. For example, when the stimulus and the response site involved the same extremity, the median amplitude of responses was larger from that extremity than from the contralateral one. Results of Wilcoxon tests indicated this was a reliable finding $(\mathrm{P}<$ 0.05 ) for the four comparisons involving both the upper and lower extremities. A particularly noteworthy finding, not reflected in the percentage data, was that the responsiveness of the palmar sites was a function of which lower extremity was stimulated. Specifically, the larger median response amplitude was associated with the palm contralateral to the stimulated foot. Inspection of the data for the individual subjects indicated that this was true in seven of the 10 cases. For the remaining individuals, one palm exhibited a consistently higher response amplitude than the other regardless of which foot was stimulated. However, the relative amplitude of the responses from the palms shifted systematically with a change in the stimulus site in a manner consistent with that exhibited by the other seven subjects.

Results obtained with the lower current levels applied during the stimulus work-up procedure were consistent with the outcomes described above. As successively higher current levels were applied to each extremity, it was noted that responses almost always occurred first from a palmar recording site-namely, from the palm contralateral to the foot being stimulated or from the palm of the stimulated hand.
Data pertaining to the level of skin conductance for each extremity were obtained by averaging values sampled immediately preceding and after presentation of the auditory stimuli. The median values were as follows: left palm, 3.9 $\mu$ mhos; right palm, $4.3 \mu$ mhos; left planta, 1.31 $\mu$ mhos; right planta, $2.20 \mu$ mhos. Except for one comparison involving one subject, the conductance level of each palmar site was higher than the level for the ipsilateral planta.

Reflex movements of the limbs and digits resulted much more consistently from plantar stimulation than from applying the stimulus to a palmar site. For all patients, stimulation of either plantar surface was associated with some degree of withdrawal movement of the stimulated extremity. The extent of withdrawal activity varied from slight dorsiflexion of the toes and ankle (four patients) to activation of the hip flexors, brisk flexion of the knee, as well as vigorous dorsiflexion of the ankles and toes (three patients). Only two patients exhibited trace movements in the leg contralateral to the one being stimulated. The latter responses were quite weak, being limited to dorsiflexion of the hallux in one case and plantar flexion of the ankle in the other. Upper extremity movements were never detected when a plantar surface was stimulated. Applying the pulse train to a palm was never associated with detectable movements of either lower extremity. For three individuals, palmar stimulation did result in flexion of the thumb and of one or more fingers of the hand being stimulated, though no movements of the contralateral upper extremity were evoked.

\section{DISCUSSION}

The analysis of skin conductance responses from the volar surfaces of patients with a chronic transection of the cervical spinal cord indicates that the reflex sympathetic outflow varies systematically depending upon the site of electrocutaneous stimulation. A general finding was that responses from the stimulated extremity occurred with a higher incidence and were of higher amplitude than responses from the opposite extremity. This proved to be the case regardless of whether the stimulus and recording sites involved the palms or the plantar surfaces. Whether the lateralization of responding reflects 
the anatomical organization of the afferent pathways or an active inhibitory process acting upon the contralateral sympathetic motoneurones remains to be assessed.

Consistent with previous findings (Fuhrer, 1971a), there was evidence of considerable intersegmental irradiation of the afferent input, exemplified by the bilateral responsiveness of the palmar sites to a pulse train applied to one lower extremity or the other. A noteworthy additional finding was that the amplitude of the palmar responses characteristically exceeded the size of the response from the plantar surface which had been stimulated. This finding is consistent with Ladpli's (1962) results based on the study of skin potential responses recorded from the chronic spinal cat. In animals with a transection of the cord between the first and second thoracic segments, electrical stimulation of the tail yielded bilaterally recorded responses from the forepaws, but little or no responding from either hindpaw. An additional outcome of the present study was that unilateral stimulation of the upper extremities was not adequate to evoke responses from either plantar surface. The differential reactivity of the upper and lower extremity recording sites may simply be indicative of a higher concentration of potentially active sweat glands on the thenar eminence of the palm than on the plantar arch. This possibility is supported by the finding that skin conductance levels, which according to Thomas and Korr (1957) are linearly related with the number of active sweat glands, were substantially higher for the palmar sites than for sites on the plantar surfaces.

The amplitude of bilaterally recorded palmar responses to lower extremity stimulation was clearly a function of the laterality of the stimulus site. Specifically, the larger of the two responses was recorded from the palm contralateral to the foot which was stimulated. It is attractive to consider the spinothalamic tract as being the principal ascending somatic pathway involved in this crossover effect. As indicated by Brodal (1969), this intraspinal pathway is supplied in part by primary dorsal root fibres which mediate sensations of cutaneous pain, and there is evidence in man that the spinothalamic fibres cross the midline shortly after their origin. An alterna- tive pathway would be a polyneuronal chain ascending within the grey matter of the cord.

Building on the work of Sherrington (1906), it has been demonstrated frequently that sympathetic reflex activity may accompany activation of spinal somatic reflexes including flexor withdrawal movements (Gellhorn, 1967). In the spinal preparation, specific parallels have been established between electrodermally monitored sudomotor responses and withdrawal activity-for example, both are susceptible to a period of spinal shock (Ladpli, 1962) and both involve $\alpha$-cutaneous fibres in their afferent pathway (Eccles and Lundberg, 1959; Holmqvist and Lundberg, 1961; Lloyd, 1962). At the same time, the present results obtained in spinal man highlight differences in the spatial patterning of segmentally mediated sudomotor and somatomotor reflexes. At the stimulus intensities used, reflex movements were limited almost exclusively to the stimulated extremity, suggesting little if any activation of alpha motoneurones supplying the contralateral limb or limbs distal to the level of afferent inflow. On the other hand, afferent input related to sudomotor discharge was much more widely generalized both laterally and longitudinally within the spinal cord, especially when the stimulus was applied to the plantar surface. The comparative analysis of these two reflex systems in spinal man requires additional investigations involving a greater diversity of stimulus sites, an even broader range of stimulus intensity, and the use of quantitative polyelectromyographic techniques to monitor the pattern of somatomotor responses.

Support for this investigation was provided by Research Grant NS07755-07 from the National Institute of Neurological Disease and Stroke and by the Rehabilitation Research and Training Center No. 4 of the Rehabilitation Services Administration. Sincere thanks are due to Mr Robert Townsend who assisted in acquiring and processing the data and Miss Gayle Birk who helped prepare the manuscript.

\section{REFERENCES}

Brodal, A. (1969). Neurological Anatomy in Relation to Clinical Medicine, 2nd edn. Oxford University Press: New York.

Eccles, R. M., and Lundberg, A. (1959). Supraspinal control of interneurones mediating spinal reflexes. Journal of Physiology, 147, 565-584.

Fuhrer, M. J. (1971a). Skin conductance responses mediated 
by the transected human spinal cord. Journal of Applied Physiology, 30, 663-669.

Fuhrer, M. J. (1971b). Analysis of electrodermal evidence for a paramedullary afferent tract in patients with a transection of the thoracic spinal cord. Journal of Neurology, Neurosurgery, and Psychiatry, 34, 281-288.

Fuhrer, M. J. (1973). Dishabituation of flexor withdrawal activity mediated by the functionally transected human spinal cord. Brain Research, 63, 93-102.

Fuhrer, M. J., and Kilbey, M. (1967). Effects of spinal-cord transection on electrodermal activity in man. Psychophysiology, 4, 176-186.

Gellhorn, E. (1967). Principles of Autonomic-Somatic Integrations: Physiological Bases and Psychological and Clinical Implications. University of Minnesota Press: Minneapolis, Minnesota.

Guttman, L., and Whitteridge, D. (1947). Effects of bladder distention on autonomic mechanisms after spinal cord injuries. Brain, 70, 361-404.

Head, H., and Riddoch, G. (1917). The automatic bladder, excessive sweating and some reflex conditions in gross injuries of the spinal cord. Brain, 40, 188-263.

Holmqvist, B., and Lundberg, A. (1961). Differential supraspinal control of synaptic actions evoked by volleys in the flexion reflex afferents in alpha motoneurones. Acta Physiologica Scandinavica, Suppl., 186, 1-51.

Ladpli, R. (1962). Galvanic skin reactions of chronic spinal cats. American Journal of Physical Medicine, 41, 15-22.
List, C. F., and Pimenta, D. (1944). Sweat secretion in man. 6. Spinal reflex sweating. Archives of Neurology and Psychiatry, 51, 501-507.

Lloyd, P. C. (1962). The classification of galvanic skin reflex afferent fibers. Proceedings of the National Academy of Sciences of the United States of America, 48, 814-817.

Richter, C. P. (1930). Galvanic skin reflex from animals with complete transection of the spinal cord. American Journal of Physiology, 93, 468-472.

Richter, C. P., and Shaw, M. B. (1930). Complete transections of the spinal cord at different levels. Archives of Neurology and Psychiatry, 24, 1107-1116.

Sherrington, C. S. (1906). The Integrative Action of the Nervous System. Yale University Press: New Haven, Connecticut.

Siegel, S. (1956). Non-parametric Statistics for the Behavioral Sciences. McGraw-Hill: New York.

Sourek, K. (1964). Die Bedeutung des galvanischen Hautreflexes für die Lokalisation von Läsionen in Nervensystem des Menschen. Acta Neurochirurgica, 11, 518-529.

Sourek, K. (1965). The Nervous Control of Skin Potentials in Man. Czechoslovak Academy of Sciences: Prague.

Thomas, A. (1921). Le Reflexe Pilomoteur. Masson: Paris.

Thomas, A., and Korr, I. M. (1957). Relationship between sweat gland activity and electrical resistance of the skin. Journal of Applied Physiology, 10, 505-510. 